\title{
Energy Determination and Gamma/Hadron Separation Using the Lateral Distribution of EAS for the 100 TeV Gamma-Ray Astronomy
}

\author{
K. Kawata*, T. K. Sako, M. Ohnishi, M. Takita \\ Institute for Cosmic Ray Research, University of Tokyo, Kashiwa, Chiba, Japan \\ E-mail: kawatadicrr.u-tokyo.ac.jp
}

\begin{abstract}
We study the energy determination of the gamma-ray induced air shower and gamma/hadron separation using the lateral distribution of extensive air shower in the energy range between $10 \mathrm{TeV}$ and $1000 \mathrm{TeV}$. For this purpose, we carry out a detailed MC simulation assuming the Tibet air shower array located in Tibet, at an altitude of $4,300 \mathrm{~m}$ above sea level. In this paper, we define $\mathrm{S} 30$, which is the particle density at $30 \mathrm{~m}$ from the air shower axis, as a new energy estimator. Using the S30, the energy resolutions are estimated to be approximately $40 \%$ at $10 \mathrm{TeV}$ and $15 \%$ at $100 \mathrm{TeV}$ in the rage of the zenith angle $\theta<20^{\circ}$. We also compare the reconstructed age distribution between the gamma-ray induced air shower and the hadronic cosmic-ray air shower. The age parameter may help to discriminate between the primary gamma rays and the hadronic cosmic rays independent of the muon information.
\end{abstract}

The 34th International Cosmic Ray Conference,

30 July- 6 August, 2015

The Hague, The Netherlands

\footnotetext{
${ }^{*}$ Speaker.
} 


\section{Introduction}

More than 100 gamma-ray sources have been detected by the Cherenkov telescopes in the energies from sub-TeV to multi-TeV. On the other hand, the extensive air shower (EAS) arrays, such as the Tibet air shower array, the ARGO-YBJ and the Milagro, have observed several gammaray sources with the wide field of view and higher energy threshold than the Cherenkov telescopes. Aiming at $100 \mathrm{TeV}$ gamma-ray astronomy, the Tibet water-Cherenkov-type muon detector (MD) array $\left(\sim 4000 \mathrm{~m}^{2}\right)$ was constructed under the Tibet air shower (AS) array $\left(\sim 37000 \mathrm{~m}^{2}\right)$, and the data taking was started in 2014 [四]. This AS+MD array will significantly improve gamma-ray sensitivity in 10-1000 $\mathrm{TeV}$ region by means of gamma/hadron separation based on counting the number of muons accompanying an air shower [ [2], [3]. In this work, we will mainly study the energy determination of gamma-ray-induced air showers in the 10-1000 TeV energy region using the lateral distribution of EAS by Monte Carlo (MC) simulation assuming the Tibet AS array. We will also show possibility of selecting gamma-ray showers from the hadron backgrounds using the age parameter of the air shower. It is noted that the muon information measured by the the MD array is not used to select gamma-ray showers in this study.

\section{Tibet Air Shower Array}

The Tibet AS array consists of 761 fast timing (FT) counters with 28 density (D) counters surrounding them [ [ 9 ]. In the inner $36,900 \mathrm{~m}^{2}$, FT counters are deployed at $7.5 \mathrm{~m}$ lattice intervals. Each of the FT counters is equipped with a fast-timing photomultiplier tube (FT-PMT) that measures up to 15 particles/counter. Among the 761 FT counters, 249 sets of counters with intervals of $15 \mathrm{~m}$ are also equipped with density photomultiplier tubes (D-PMT) of wide dynamic range that measure up to 5000 particles/counter in addition to the FT-PMTs.

Each counter has a plastic scintillator plate of $0.5 \mathrm{~m}^{2}$ in area and $3 \mathrm{~cm}$ in thickness. A lead plate $0.5 \mathrm{~cm}$ thick is put on the top of each counter in order to increase the counter's sensitivity by converting the photons in an electromagnetic shower into electron-positron pairs. The recording of signals is made for time and charge information for the FT-PMTs, but only for the charge information for the D-PMTs. The D counters surrounding the inner array are also equipped with both FT-PMTs and D-PMTs, where only the charge information of both PMTs is recorded. An event trigger signal is issued when any four-fold coincidence occurs in FT counters recording more than 0.6 particles.

\section{MC Simulation}

The performance of the Tibet AS array is studied by a full MC simulation based on the Corsika code [ []] for air shower event generation and the GEANT4 code [焑] for the response of each scintillation counter, respectively. The air showers of primary gamma rays and cosmic rays are generated by the Corsika with EPOS LHC $[\square]$ as a hadronic interaction model assuming the following spectra. The primary gamma-ray spectrum is the simple power-law spectrum above $0.3 \mathrm{TeV}$ with an index -2.0. The primary cosmic-ray spectrum and its mass composition are sampled from the energy spectrum made by mainly using the direct observational data above $0.3 \mathrm{TeV}$. These simulated air 


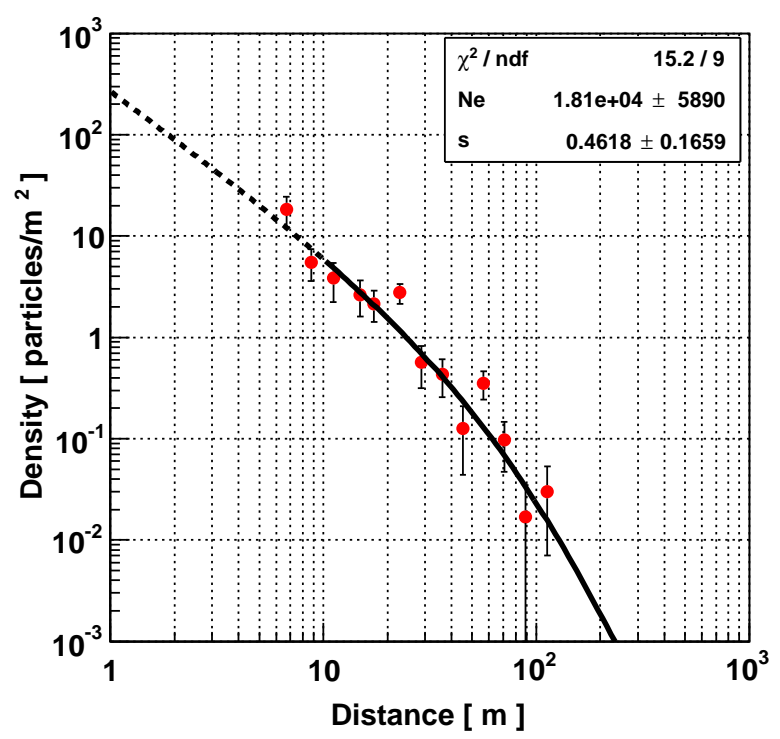

Figure 1: Lateral distribution of the $18 \mathrm{TeV}$ gamma-ray event generated by the $\mathrm{MC}$ simulation assuming the Tibet AS array. The solid curve shows fitted result by the NKG function further than $10 \mathrm{~m}$. The dashed curve is an extrapolation of the fitted NKG function within $10 \mathrm{~m}$.

shower events are fed into the detector simulation based on the GEANT4 code, and are analyzed in the same way as the experimental data to reconstruct the energy and the arrival direction.

\section{Analysis}

The primary gamma-ray energy is estimated from the lateral distribution of particle density in an air shower. To estimate air shower size (the total number of particles in an air shower at the altitude of our site), we fitted the lateral distribution of particle density measured by the Tibet AS array using the following Nishimura-Kamata-Greisen (NKG) function [ [8, Q].

$$
\rho(r)=\frac{N_{\mathrm{e}}}{r_{\mathrm{m}}^{2}} \frac{\Gamma(4.5-s)}{2 \pi \Gamma(s) \Gamma(4.5-2 s)}\left(\frac{r}{r_{\mathrm{m}}}\right)^{s-2}\left(1+\frac{r}{r_{\mathrm{m}}}\right)^{s-4.5},
$$

where $r$ denotes the distance from air shower axis, free parameters $N_{\mathrm{e}}$ and $s$ denote the air shower size and the age of the air shower, respectively, fixed parameter $r_{m}$ (Moliere unit) is set to be $130 \mathrm{~m}$. The counters located closer than $10 \mathrm{~m}$ from the air shower axis are not used. The FT (D) counters detecting more than 15 (5000) particles are not used, because the PMT linearity fails. Figure $\mathbb{W}$ shows typical lateral distribution of the $18 \mathrm{TeV}$ gamma-ray event generated by the MC simulation. The zenith angle of this event is $10.3^{\circ}$, and the number of hit counters is 39 .

The primary cosmic-ray energy is traditionally estimated from the reconstructed air shower size $\left(N_{\mathrm{e}}\right)$ using the NKG function. On the other hand, in the ultra-high-energy cosmic-ray field with $E>\sim 10^{18} \mathrm{eV}$ observed by a widely spread array ( $>1 \mathrm{~km}$ spacing), the particle density at a specific distance from the shower axis is well established as an energy estimator, since this depends weakly on variation in the interaction models, fluctuation in shower development, and the primary 


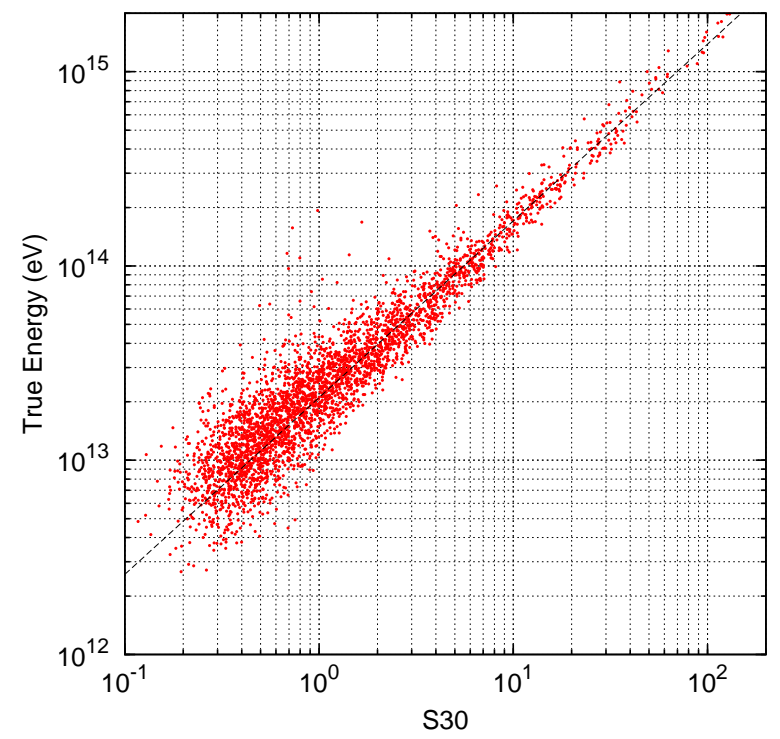

Figure 2: Correlation between the primary gamma-ray energy and S30 calculated from the lateral fitting by the MC simulation assuming the Tibet AS array. The zenith angle of the air shower $\theta$ is smaller than $20^{\circ}$.

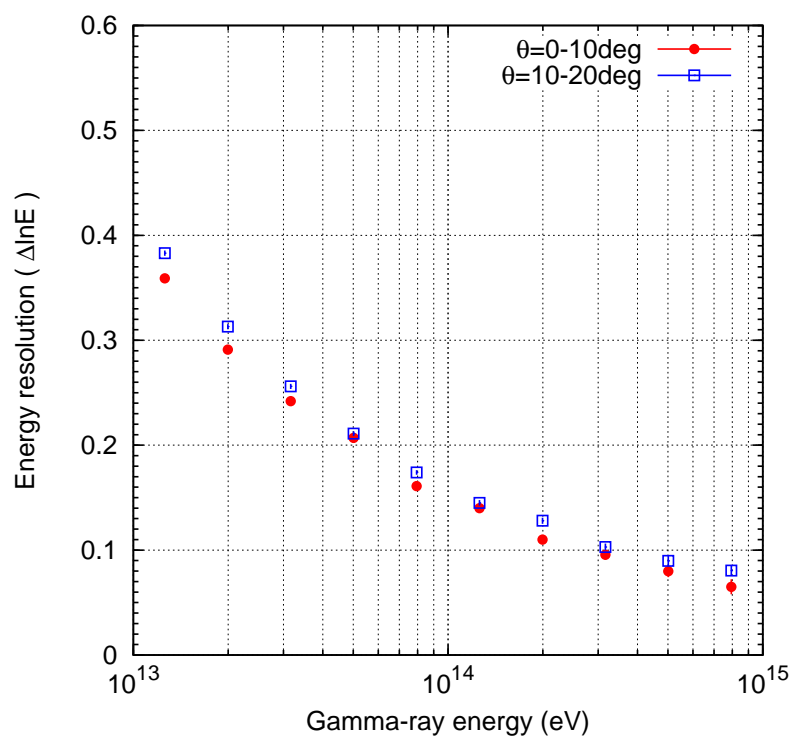

Figure 3: Energy resolution $(\Delta \ln E)$ vs primary gamma-ray energy generated by the MC simulation assuming the Tibet AS array. $\Delta \ln E$ is the standard deviation of the logarithmic Gaussian function. The red points and blue squares show in case of the zenith angle $0^{\circ}<\theta<10^{\circ}$ and $10^{\circ}<\theta<20^{\circ}$, respectively.

mass [प]]. For example, the Telescope Array experiment have adopted S800, which is the particle density at a lateral distance of $800 \mathrm{~m}$ from the core, as an energy estimator [U]]. In this paper, we define an air-shower parameter $\mathrm{S} 30$, which is the particle density at $30 \mathrm{~m}$ from the air shower axis $(\rho(30)$ in Equ.4.1), as a new energy estimator. 


\section{Results and Discussions}

The event selections of the MC events are the following criteria: (1) each shower event should fire 16 or more FT counters that have each recorded 1.25 or more particles; (2) The air shower core should be inside of the array (3) the zenith angle of the event arrival direction should be less than $20^{\circ}$.

Figure $\square$ shows the correlation between the generated gamma-ray energy $\left(E_{\mathrm{GEN}}\right)$ and S30 calculated from the lateral fitting. The reconstructed gamma-ray energy $\left(E_{\mathrm{REC}}\right)$ is directly derived from this correlation. The $\mathrm{S} 30$ is the better estimator than $N_{\mathrm{e}}$ (shower size), since this is weakly affected by the fluctuation in the air shower. Figure 3 shows the energy resolution as a function of the primary gamma-ray energy. The resolutions are estimated by fitting $\ln \left(E_{\mathrm{REC}} / E_{\mathrm{GEN}}\right)$ distribution by the logarithmic Gaussian function. The vertical axis means the standard deviation $(\Delta \ln E)$ of the logarithmic Gaussian function. The energy resolutions for $100 \mathrm{TeV}$ and $10 \mathrm{TeV}$ gamma ray are estimated to be $\Delta \ln E=0.15$ and 0.38 , which correspond to $(+16 /-14) \%$ and $(+46 \% /-32) \%$, respectively.

The age parameter of the NKG function has been utilized for discriminating gamma-ray showers from the hadronic cosmic-ray showers above a few hundred $\mathrm{TeV}$ [12, [13]. Figure 4 shows the reconstructed age distributions of gamma-ray and hadronic cosmic-ray showers with $E_{\mathrm{REC}}>50 \mathrm{TeV}$, $\theta<20^{\circ}$ by the MC simulation assuming the Tibet AS array. The average age of gamma-ray showers shows smaller than that of cosmic-ray showers, since the longitudinal development of the gamma-ray air showers, which are dominated by pure electromagnetic cascade, is faster than that of the cosmic-ray showers. The strong discrimination power can not be expected, however, the age parameter may help to discriminate between them independent of the muon information.

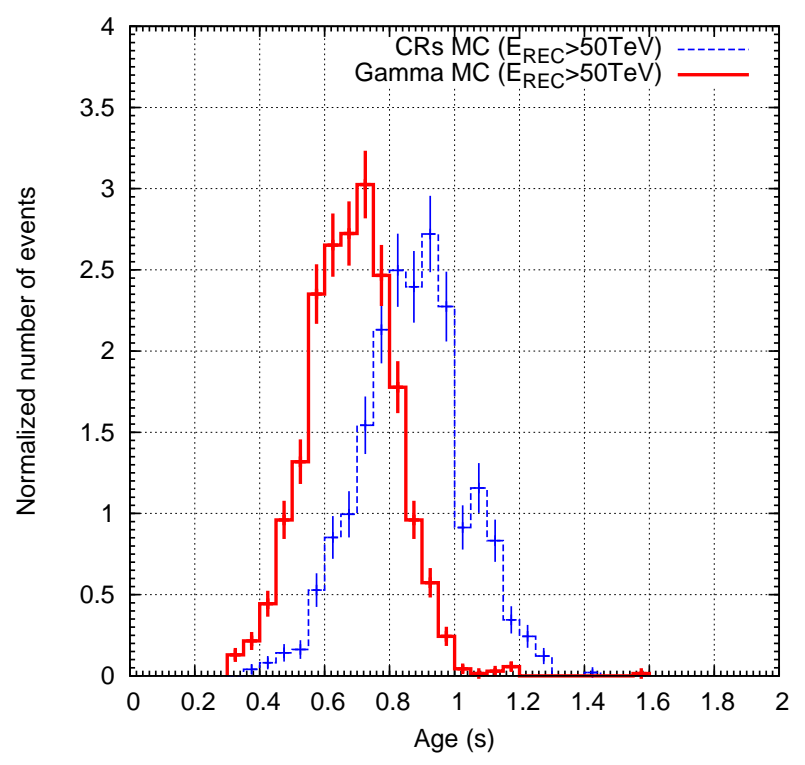

Figure 4: Reconstructed age distribution with $E_{\mathrm{REC}}>50 \mathrm{TeV}, \theta<20^{\circ}$ by the $\mathrm{MC}$ simulation assuming the Tibet AS array. The solid red and dashed blue histograms show the gamma-ray induced air shower and hadronic cosmic-ray induced air shower, respectively. The fitting parameter range of the age is set to be $0.3<s<1.6$. 


\section{Summary}

We study the lateral distribution of the particle density in the gamma-ray induced air shower by a MC simulation assuming the Tibet AS array in the energy range between $10 \mathrm{TeV}$ and $1000 \mathrm{TeV}$. Using the new energy estimator S30, the energy resolutions for the gamma rays are estimated to be approximately $40 \%$ at $10 \mathrm{TeV}$ and $15 \%$ at $100 \mathrm{TeV}$ at the zenith angle $\theta<20^{\circ}$. We also compare the reconstructed age distribution between the gamma-ray induced air shower and the hadronic cosmic-ray induced air shower. The age parameter may help to discriminate between the primary gamma rays and the hadronic cosmic rays independent of the muon information. These studies will be applied to the real data collected by the Tibet AS+MD array since 2014 .

\section{Acknowledgments}

We would like to thank the Tibet AS $\gamma$ collaborators for fruitful discussions.

\section{References}

[1] M. Amenomori, et al., "Tibet AS+MD Project: progress report 2015", these proceedings (2015)

[2] M. Amenomori, et al., Ap\&SS, 309, 435 (2007)

[3] T. K. Sako, K. Kawata, M. Ohnishi, A. Shiomi, M. Takita, and H. Tsuchiya, Astropart. Phys., 32, 177 (2009)

[4] M. Amenomori, et al., ApJ, 678, 1165 (2008)

[5] D. Heck, J. Knapp, J. N. Capdevielle, G. Shatz, and T. Thouw, Report FZKA 6019, Karlsruhe: Forschungszentrum Karlsruhe (1998)

[6] S. Agostinelli et al., Nucl. Instrum. Methods Phys. Res. A 506, 250 (2003)

[7] T. Pierog, lu. Karpenko, J. M. Katzy. E. Yatsenko, and K. Werner, arXiv:1306.0121 (2013)

[8] K. Kamata and J. Nishimura, Prog. Theor. Phys., 6, 93 (1958)

[9] K. Greisen, Ann. Rev. Nucl. Part. Sci., 10, 63 (1960)

[10] M. Takeda, et al., Phys. Rev. Lett., 81, 1163 (1998)

[11] T. Abu-Zayyad, et al., ApJ, 768, L1 (2013)

[12] R. K. Dey and A. Bhadra, Astropart. Phys., 44, 68 (2012)

[13] A. Tapia, et al., the proceedings of the ICRC 2013, arXiv:1309.3536 (2013) 